\title{
Maturidade em Gerenciamento de Projetos Estratégicos: fatores de impacto em uma instituição pública de educação
}

\author{
Maturity in Strategic Project Management: \\ impact factors in a public education institution
}

\author{
Roger Campo Dall'Orto Guimarães ${ }^{1}$ \\ Sérgio Augusto Pereira Bastos ${ }^{2}$
}

\begin{abstract}
Resumo
O presente estudo tem como objetivo identificar os fatores que influenciam a percepção de maturidade sobre o gerenciamento de projetos estratégicos. Para tal, foi realizado um estudo de caso, de natureza exploratória, de corte transversal, com uma amostra de 142 gerentes e membros de equipes de projetos estratégicos do Instituto Federal do Espírito Santo (IFES). Os dados foram coletados por meio de questionários semiestruturados, complementados por análise factual e documental. Os dados foram tratados de forma quantitativa e qualitativa. Os resultados mostraram que as variáveis que influenciam a percepção de maturidade em gerenciamento de projetos no Instituto foram o gerenciamento do tempo, custos, comunicações, partes interessadas e aquisições, assim como o grau de instrução do profissional e a estrutura organizacional com um gerente de projeto mais dedicado e com o apoio da alta administração. Os resultados, embora não possam ser generalizados, indicam caminhos para o direcionamento de políticas e incentivos para aumentar o desempenho de projetos estratégicos em instituições públicas de educação.
\end{abstract}

Palavras-chave: Maturidade em Gerenciamento de Projetos; Projetos Estratégicos; Instituição Pública de Educação.

\footnotetext{
Mestre em Administração de Empresas - FUCAPE Business School. Rio de Janeiro, RJ - Brasil. ORCID: https://orcid.org/0000-0002$\underline{9909-5251}$ | rogercamposguimaraes@gmail.com

2 Doutor em Administração de Empresas - Pontifícia Universidade Católica do Rio de Janeiro - PUC-Rio; Professor Associado da FUCAPE Business School. Rio de Janeiro, RJ - Brasil. ORCID: https://0000-0002-5697-3624 | sbastos@,fucape.br
}

Como citar:

Guimarães, R. C. D., \& Bastos, S. A. P. (2020). Maturidade em Gerenciamento de Projetos Estratégicos: fatores de impacto em uma instituição pública de educação. Revista Inovação, Projetos e Tecnologias, 8(1), 51-69. https://doi.org/10.5585/iptec.v8i1.15875 


\begin{abstract}
The present study aims to identify the factors that influence the perception of maturity over strategic projects management. A cross-sectional exploratory case study was carried out with a sample of 142 managers and members of strategic projects teams from the Federal Institute of Espírito Santo (IFES). The data were collected through semi structured questionnaires, complemented with factual and documentary analysis. Data was treated quantitative and qualitatively. The results showed that the variables that influence the perception of maturity in project management at the Institute were time management, costs, communications, stakeholders and acquisitions, as well as the degree of the managers' professional education and the organizational structure with a full-time project manager supported by senior management. The results, although they cannot be generalized, point out ways to guide policies and incentives to increase the performance of strategic projects in public education institutions.
\end{abstract}

Keywords: Maturity in Project Management; Strategic Projects; Public Education Institution.

\title{
1 Introdução
}

De forma geral, no âmbito das organizações, desenvolver capacidade de gerar resultados é um desafio crescente. No setor privado, a constante exigência por uma governança corporativa tem aumentado a demanda por parte dos acionistas para uma maior prestação de contas, transparência e capacidade de implementar a estratégia (Crawford \& Helm, 2009). No setor público, esta tendência encontra-se sob pressão semelhante, com ainda maior destaque para a satisfação das inúmeras partes interessadas (stakeholders), demandando transparência e responsabilidade à aplicação de políticas com comprovações de resultados e adaptação às mudanças (Edwards \& Clough, 2005).

Nesse contexto, o gerenciamento de projetos tem se apresentado como um conjunto de processos capazes de auxiliar a implementação de estratégias, conferindo valor em sistemas de entregas e colaborando, assim, para a realização de objetivos organizacionais (Srivannaboon, 2006). Mais especificamente, a Maturidade em Gerenciamento de Projetos (MGP), como descrita por Kerzner e Kerzner (2017), é um importante elemento para a elaboração e implementação do planejamento estratégico, sendo composta do desenvolvimento de sistemas e processos repetitivos que proporcionam uma alta probabilidade de sucesso de projetos. Há, porém, que destacar que diversos autores questionam a idealização e a composição das diversas metodologias de apuração de maturidade projetos (Pasian, Willians, \& Alameri, 2012; Wheatley, 2007) e propõem abordagens mais integradas, como Görög (2016).

As organizações públicas da administração direta e indireta estão entre as que apresentam menores índices em maturidade de projetos (Prado \& Archibald, 2009), índices provenientes de diferentes metodologias e que variam conforme a indústria (Carvalho, Patah, \& Bido, 2015; CookeDavies \& Arzymanow, 2003; Grant \& Pennypacker, 2006; Ibbs \& Kwak, 2000; Prado \& Archibald, 2009). No entanto, o setor de serviços educacionais tem sido negligenciado nas pesquisas sobre a implementação de projetos estratégicos e o papel que o grau de maturidade no gerenciamento de projetos desempenha no sucesso dos projetos e, consequentemente, no desempenho esperado pelo planejamento estratégico. Além disso, há pouca evidência empírica de que modelos de qualificação e diagnóstico da maturidade no gerenciamento de projetos leve a melhorias organizacionais, de desempenho e de geração de valor (Mullaly, 2014).

Com isso, o problema que se coloca nessa pesquisa é como contribuir para que o planejamento estratégico, que aponta para o desenvolvimento de projetos estratégicos, seja bemsucedido à luz do processo de gerenciamento de projetos das organizações, com ênfase para as instituições públicas de ensino.

Logo, em um cenário em que a capacidade de uma organização em obter sucesso na implementação de seu planejamento estratégico pode estar relacionada com sua maturidade em gerenciamento de projetos, esse trabalho tem como objetivo identificar os fatores que influenciam a 
percepção de maturidade sobre o gerenciamento de projetos. Para isso, o objeto de estudo foi o Instituto Federal do Espírito Santo (IFES), organização com 22 campi, um centro de formação a distância, aproximadamente 36 mil alunos e uma diversidade de projetos em andamento decorrentes de um planejamento estratégico que foi primeiramente formalizado para o período 2014-2018, juntamente com a implementação de um processo de gerenciamento de projetos estratégicos. Ou seja, o IFES inclui-se na problemática destacada anteriormente.

A motivação deste trabalho, em termos de contribuição à gestão das organizações, particularmente educacionais e no setor público, vem da necessidade de conferir maior eficiência sobre recursos cada vez mais escassos, principalmente, quando se trata de projetos, sejam de investimentos ou de natureza operacional. Os determinantes dessas avaliações poderão nortear não só o IFES, mas também outras organizações, de ensino ou não, púbicas ou privadas, e de outros Institutos que integram a Rede Federal de Ensino para direcionar suas práticas de gerenciamento de projetos. Não menos importante é a contribuição teórica ao campo de gerenciamento de projetos, no que tange aos modelos de maturidade e às estruturas de gestão de projetos.

\section{Referencial teórico}

\subsection{Fatores críticos de sucesso no gerenciamento de projetos}

A publicação do guia de conhecimento para o gerenciamento de projetos, o Project Management Book of Knowledgment (PMBOK), pelo Project Management Institute (PMI), em 1996, foi um grande marco para evolução do conhecimento e disseminação de práticas do tema. O gerenciamento de projetos era, então, estruturado em nove áreas de conhecimento. O guia, em sua quinta edição, está estruturado em 10 áreas do conhecimento e 47 processos de gerenciamento de projetos (PMI, 2013), como mostra a Tabela1. Há que destacar que uma sexta versão do PMBOK está disponível desde 2017. No entanto, o objeto de estudo em questão, o IFES, utiliza a quinta versão, de 2013, como referência para a metodologia de gerenciamento de projetos elaborada e implementada na Instituição, na qual foram treinados os gestores de projetos.

Tabela 1: Áreas do conhecimento e processos de gestão de projetos do PMBOK

\begin{tabular}{cl}
\hline $\begin{array}{c}\text { Áreas do } \\
\text { conhecimento }\end{array}$ & \multicolumn{1}{c}{ Processos } \\
\hline Integração & $\begin{array}{l}\text { Desenvolver o termo de abertura do projeto; desenvolver o plano de gerenciamento do } \\
\text { projeto; orientar e gerenciar o trabalho do projeto; monitorar e controlar o trabalho do } \\
\text { projeto; realizar o controle integrado de mudanças; e encerrar o projeto ou fase. }\end{array}$ \\
\hline Escopo & $\begin{array}{l}\text { Planejar o gerenciamento do escopo; coletar os requisitos; definir o escopo; criar a } \\
\text { EAP; validar o escopo; e controlar o escopo. }\end{array}$ \\
\hline Tempo & $\begin{array}{l}\text { Planejar o gerenciamento do cronograma; definir as atividades; sequenciar atividades; } \\
\text { estimar os recursos das atividades; estimar as durações das atividades; desenvolver o } \\
\text { cronograma; e controlar o cronograma. }\end{array}$ \\
\hline Custos & $\begin{array}{l}\text { Planejar o gerenciamento dos custos; estimar custos; determinar o orçamento; e } \\
\text { controlar os custos. }\end{array}$ \\
\hline
\end{tabular}




\begin{tabular}{cl}
\hline Qualidade & $\begin{array}{l}\text { Planejar o gerenciamento da qualidade; realizar a garantia de qualidade; e controlar a } \\
\text { qualidade. }\end{array}$ \\
\hline $\begin{array}{c}\text { Recursos } \\
\text { Humanos }\end{array}$ & $\begin{array}{l}\text { Planejar o gerenciamento dos recursos humanos; mobilizar a equipe do projeto; } \\
\text { desenvolver a equipe do projeto; e gerenciar a equipe do projeto. }\end{array}$ \\
\hline Comunicação & $\begin{array}{l}\text { Planejar o gerenciamento das comunicações; gerenciar as comunicações; controlar as } \\
\text { comunicações. }\end{array}$ \\
\hline Riscos & $\begin{array}{l}\text { Planejar o gerenciamento dos riscos; identificar os riscos; realizar a análise qualitativa } \\
\text { dos riscos. Realizar a análise quantitativa dos riscos; planejar as respostas aos riscos; e } \\
\text { controlar os riscos. }\end{array}$ \\
\hline Aquisições & $\begin{array}{l}\text { Planejar o gerenciamento das aquisições; conduzir as aquisições; controlar as } \\
\text { aquisições; e encerrar as aquisições. }\end{array}$ \\
\hline Partes & $\begin{array}{l}\text { Identificar partes interessadas; planejar o gerenciamento das partes interessadas; } \\
\text { gerenciar o envolvimento das partes interessadas; e controlar o envolvimento das } \\
\text { partes interessadas. }\end{array}$
\end{tabular}

Fonte: PMI (2013), em tradução livre dos autores.

Estudos mais recentes exploraram os componentes do gerenciamento de projetos necessários para obter sucesso em seus empreendimentos. Esses componentes, em grande parte, estão relacionados com as áreas do conhecimento e os processos de gerenciamento de projetos preconizados pelo PMI. Em uma pesquisa empírica com mais de 70 grandes organizações nacionais e multinacionais nos Estados Unidos foi identificado que os fatores determinantes para o sucesso em projetos estavam relacionados à gestão de riscos, responsabilidades organizacionais do projeto, controle de mudanças de escopo e manutenção de integridade das linhas de base (Cooke-Davies, 2002):

Em organizações do Reino Unido, liderança e equipe de gerenciamento, política e estratégia, parcerias e processos do ciclo de vida do projeto foram relacionados como fatores críticos para o bom desempenho no gerenciamento de projetos (Bryde, 2003). Estas evidências foram resultantes da proposição de seu modelo para avaliar o desempenho em gerenciamento de projetos ligado ao conceito de gestão da qualidade.

Abdullah e Ramly (2006) elaboraram um estudo para identificar os vários componentes de sucesso de projetos. O estudo foi realizado entre gerentes de projeto e identificou que os critérios de sucesso (o que) de um projeto podem ser resumidos como tempo, custo, qualidade e valorização dos stakeholders. Já os fatores de sucesso (como) foram a gestão humana, processos e organização, técnicas e contratos, liderança e equipe, gerente de projeto, gerenciamento de stakeholders, planejamento, programação, organização, controle e monitoramento, recursos financeiros e gestão da qualidade.

No Brasil, um recente estudo de Berssaneti e Carvalho (2015) realizou uma pesquisa tipo survey com 336 profissionais da área de gestão de projetos em diferentes setores da economia e mostrou o significativo relacionamento entre maturidade em gestão de projetos e o desempenho do projeto em termos de tempo, custo e requisitos do projeto. Revelou, também, a relevância de ter um gerente de projeto dedicado para o sucesso dos projetos em termos de prazo, e por fim o apoio da alta gestão para o sucesso dos projetos em termos de prazo e no atendimento aos requisitos do projeto. 
Desta forma, as organizações devem melhorar as suas capacidades, desenvolvendo-se para níveis de maturidade mais elevados nas áreas de conhecimento determinantes em sua atuação, ou seja, priorizando aquelas áreas que mais impactam significativamente o desempenho de seu negócio, uma vez que não há fatores críticos de sucessos comuns a todos os projetos (Cooke-Davies, 2002; Ika, Diallo, \& Thullier, 2012).

\subsection{Maturidade em gerenciamento de projetos}

Avaliar as capacidades atuais e auxiliar a organização alcançar melhor desempenho no gerenciamento de projetos é o papel dos modelos de maturidade de gerenciamento de projetos (Meng, Sun, \& Jones, 2011)__Organizações maduras, como mencionado por Cooke-Davies (2004), são capazes de gerir seus projetos de forma eficaz e demonstram melhoria consistente em seu desempenho.

Os modelos de maturidade em gestão de projetos têm como objetivo avaliar o contexto e os processos de gerenciamento de projetos dentro das organizações. Os resultados dessas avaliações devem apoiar as ações estratégicas que orientam o gerenciamento de portfólio, programas e projetos no sentido de aumentar o grau de sucesso dos empreendimentos (Berssaneti \& Carvalho, 2015; Neves, Trentim, \& Nunes, 2014).

A atenção aos modelos de maturidade de gestão de projetos ganha força a partir da década de 1990. Desde então, houve uma propagação no desenvolvimento de Modelos de Maturidade em Gestão de Projetos (MMGP) e mais de 30 (trinta), com abordagens diferenciadas, podem ser identificados na literatura. Desses, valem ser destacados, em função da popularidade de uso e presença constante na literatura de gerenciamento de projetos, os seguintes: 1) CMMI - Capability Maturity Model Integration: desenvolvido pelo Software Engineering Institute (SEI), da Carnegie Mellon University em 1986; 2) KPMMM - Kerzner Project Management Maturity Model: desenvolvido por Harold Kerzner em 1998; 3) PMMM - Project Management Maturity Model: desenvolvido por Crawford, da PM Solutions, em 2001; 4) MMGP - Modelo de Maturidade em Gerenciamento de Projetos: desenvolvido por Darci Prado em 2002; e 5) OPM3 - Organizational Project Management Maturity Model: desenvolvido pelo PMI em 2003.

O Project Management Maturity Model (PMMM), desenvolvido por Crawford (2001) é o escolhido como referência para o presente estudo, já que utiliza as áreas do conhecimento descritas no Guia de Conhecimentos de Gerenciamento de Projetos - PMBOK (PMI, 2013) para avaliar a maturidade da organização e assim, orientá-la a buscar excelência na gestão de projetos. O PMBOK, por sua vez, é a opção metodológica na organização objeto deste estudo.

Como consequência da evolução no grau de maturidade em gerenciamento de projetos, há maior capacidade de alcançar os objetivos estratégicos da organização. O PMMM indica o grau de maturidade em que a organização se encontra em uma escala de cinco níveis: nível 1 - processo inicial; nível 2 - processos e padrões estruturados; nível 3 - padrões de organização e processo institucionalizado; nível 4 -processo gerenciado; e nível 5-processo otimizado. Na Tabela 2 as características de cada nível são relacionadas.

O modelo para medir a maturidade em gerenciamento de projetos deve apontar um caminho lógico para o desenvolvimento progressivo do gerenciamento de projetos na organização e contribuir com o planejamento estratégico da organização (Crawford \& Helm, 2009). Dentre outros existentes na literatura, o PMMM é o modelo escolhido para avaliar a maturidade em gestão de projetos no IFES.

Tendo em vista os estudos antecessores, os fatores que impactam o sucesso no gerenciamento de projetos são representados pelas 10 áreas do conhecimento expressas no PMBOK (PMI, 2013). 
Adicionalmente, são consideradas as variáveis demográficas representativas da a percepção da maturidade institucional no gerenciamento de projetos.

Tabela 2: Modelo PMMM - Grau de maturidade e suas características

\begin{tabular}{|c|c|c|c|c|}
\hline Nível 1 & Nível 2 & Nível 3 & Nível 4 & Nível 5 \\
\hline $\begin{array}{l}\text { Consciência de } \\
\text { gestão }\end{array}$ & $\begin{array}{c}\text { Processos básicos, } \\
\text { utilizados apenas } \\
\text { em grandes projetos } \\
\text { com alta } \\
\text { visibilidade }\end{array}$ & $\begin{array}{l}\text { Todos os processos } \\
\text { padronizados para } \\
\text { todos os projetos e } \\
\text { mais de um foco } \\
\text { institucional }\end{array}$ & $\begin{array}{c}\text { Gestão com visão } \\
\text { holística e utiliza } \\
\text { dados para tomar } \\
\text { decisões }\end{array}$ & $\begin{array}{c}\text { Existência de } \\
\text { processos para } \\
\text { medir a eficácia e } \\
\text { eficiência do } \\
\text { projeto }\end{array}$ \\
\hline $\begin{array}{c}\text { Processos existentes } \\
\text { executados } \\
\text { aleatoriamente }\end{array}$ & $\begin{array}{l}\text { Gestão apoia e } \\
\text { incentiva o uso }\end{array}$ & $\begin{array}{c}\text { Gestão } \\
\text { institucionalizou } \\
\text { processos de gestão } \\
\text { de projeto e possui } \\
\text { coleta informal de } \\
\text { práticas }\end{array}$ & $\begin{array}{c}\text { Gestão engajada no } \\
\text { gerenciamento do } \\
\text { portfólio }\end{array}$ & $\begin{array}{c}\text { Processos em vigor } \\
\text { para melhorar o } \\
\text { desempenho do } \\
\text { projeto }\end{array}$ \\
\hline- & $\begin{array}{c}\text { Misto de } \\
\text { informações de } \\
\text { nível intermediário } \\
\text { e resumo }\end{array}$ & $\begin{array}{c}\text { Informações } \\
\text { resumidas, } \\
\text { detalhadas e análise } \\
\text { informal do } \\
\text { desempenho do } \\
\text { projeto }\end{array}$ & $\begin{array}{c}\text { Processos } \\
\text { integrados com os } \\
\text { processos } \\
\text { institucionais }\end{array}$ & $\begin{array}{l}\text { Gestão centra-se na } \\
\text { melhoria contínua }\end{array}$ \\
\hline- & $\begin{array}{c}\text { Estimativas e } \\
\text { cronogramas com } \\
\text { base no } \\
\text { conhecimento de } \\
\text { especialistas e } \\
\text { ferramentas } \\
\text { genéricas }\end{array}$ & $\begin{array}{c}\text { Estimativas e } \\
\text { cronogramas com } \\
\text { base nos padrões do } \\
\text { negócio e } \\
\text { especificidades } \\
\text { institucionais }\end{array}$ & $\begin{array}{l}\text { Análise sólida do } \\
\text { desempenho dos } \\
\text { projetos e exigência } \\
\text { de conformidades }\end{array}$ & - \\
\hline- & - & - & $\begin{array}{c}\text { Estimativas e } \\
\text { cronogramas com } \\
\text { base nas } \\
\text { especificidades } \\
\text { institucionais }\end{array}$ & - \\
\hline
\end{tabular}

Fonte: Crawford (2001).

O modelo proposto busca determinar o grau em que as dez áreas do conhecimento do gerenciamento de projetos são percebidas e têm sido adotadas pelos gerentes e equipes de projetos estratégicos do IFES, avaliar a percepção do nível de maturidade em gerenciamento de projetos e, por fim, identificar quais destas variáveis são determinantes na percepção de maturidade em gerenciamento de projetos.

\section{Metodologia}

A presente pesquisa pode ser classificada como descritiva, contém cunho quantitativo e qualitativo e trata-se, em termos metodológicos, de um estudo de caso. Embora os dados coletados buscassem cobrir um período de pouco mais de 3 anos (2014 a 2016), já que foram obtidos em 
meados de 2017, tendo como foco os projetos relativos ao planejamento estratégico 2014-2018, o estudo não pode ser considerado um estudo longitudinal, mas sim de corte transversal.

A população da pesquisa foi composta por todos os funcionários do IFES envolvidos nos projetos decorrentes do planejamento estratégico 2014-2018. A amostra foi composta por gerentes e membros de equipes de projetos estratégicos que responderam ao questionário semiestruturado. Ao todo, 142 questionários foram respondidos, sendo distribuídos pelos 22 campi e centro de formação a distância do IFES. Este patamar representa $49,31 \%$ do total de 288 líderes e membros de projetos nomeados por portarias oficiais.

A taxa de resposta meta foi calculada a partir da fórmula para determinação do tamanho da amostra para variáveis de escala de intervalo em populações pequenas de Rea e Parker (2000):

$$
n=\frac{z^{2} \cdot s^{2}}{C i^{2}+z^{2} \cdot s^{2} / n-1}
$$

Onde:

$\mathrm{Z}_{\alpha}=$ transformada $\mathrm{Z}$ para o nível de confiança desejado

$\mathrm{s}=$ desvio padrão da população

$\mathrm{C}_{\mathrm{i}}=$ intervalo de confiança em termos de escala de intervalos

$\mathrm{n}=$ tamanho da população

Admitiu-se, simplificadamente, que a população é homogênea, em termos de percepção, não existindo disparidades nem em função da área a que cada pessoa pertence, nem em função de seu nível hierárquico. Para a seleção dos sujeitos admitiu-se o intervalo de confiança de $99 \%(Z=2,575)$ e um erro de 0,2 , considerando-se as escalas de intervalo igual a 1 . Com relação ao desvio padrão da população, estimou-se em 0,833 , a partir de 5 intervalos de escala e seis desvios padrão para a distribuição de toda a população. A escala Likert de intervalo 1 a 5 foi a única utilizada. Com isso, tem-se que o número mínimo de respostas deveria ser de 82 pessoas. Como foram recebidos 142 questionários preenchidos $(49,31 \%$ de taxa de resposta), o intervalo de confiança e o erro máximo desejados foram atingidos e os resultados podem ser generalizados para o IFES.

O método do estudo de caso é adequado quando envolve um fenômeno contemporâneo a observado sem que sejam definidas fronteiras entre o fenômeno e seu contexto (Yin, 2015), sendo um método amplamente utilizado na pesquisa em administração (Rementyi, Willams, Money, \& Swartz, 1998). Sempre deve estar voltado para uma replicação futura, já que seus resultados não podem ser generalizados per se (Eisenhardt, 1989). Ainda segundo Yin (2015), a investigação de um estudo de caso deve se basear em várias fontes de evidência, com os dados precisando convergir a partir do levantamento de percepções e da investigação documental e de fatos.

Nas investigações documentais foram identificadas as portarias de nomeações dos membros da equipe, os termos de abertura dos projetos com a assinatura do patrocinador local do projeto, documentos do Microsoft Project e Excel para monitorar o status da execução do projeto e confrontado com o planejamento do programa ao qual o projeto é subordinado, assim como arquivos específicos dos projetos.

Foi realizado um levantamento percepções com a aplicação de questionário semiestruturado, composto por perguntas abertas e fechadas. As perguntas abertas permitiram identificar as barreiras e facilidades encontradas durante a gestão dos projetos. Já a parte do questionário contendo perguntas estruturadas foi tratada de forma quantitativa. O questionário foi aplicado de forma presencial aos 
líderes e membros das equipes dos projetos estratégicos do IFES. Foram impressos com páginas coloridas para atrair e motivar os participantes a preenchê-lo.

O questionário, em sua parte estruturada, foi composto por escalas validadas por Rasid, Ismail, Mohammad e Long (2014). Também foram obtidas informações sobre perfil demográfico da amostra e entendimento de importância, adoção e percepção do nível de maturidade nas áreas do conhecimento de gerenciamento de projetos. Um pré-teste foi realizado com 10 respondentes, sendo requeridas algumas adaptações textuais para melhor atender à natureza de instituição de ensino pública.

Para compor a nota de cada área do conhecimento, nas perguntas objetivas, foi extraída a média das respostas dos processos que compõem cada área. Foi utilizada escala Likert de 5 pontos da seguinte forma: 5 - concordo plenamente, 4 - concordo, 3 - neutro, 2 - discordo e 1 - discordo plenamente.

Os dados da parte estruturada do questionário foram estatisticamente analisados e interpretados com auxílio do software EViews. Desta forma, um modelo empírico que mensura a maturidade em gerenciamento de projetos do Instituto na percepção dos envolvidos no projeto pôde ser aplicado. Esta representação relaciona as áreas do conhecimento determinantes na compreensão da maturidade em gerenciamento de projetos, fruto dos resultados da regressão linear múltipla suportada no método de estimação stepwise, nos testes de validade ANOVA, de aderência à normalidade, aleatoriedade e homocedasticidade.

O modelo empírico da regressão tem como variável dependente o nível de maturidade do Instituto em gerenciamento de projetos percebido pelo indivíduo i, $\alpha$ indica o intercepto, os X's são as variáveis independentes, os $\beta$ 's são constantes a serem estimadas e $\varepsilon$ o erro de estimativa. Assim o modelo utilizado foi o seguinte:

$$
\begin{gathered}
\mathrm{Y}_{\mathrm{i}}=\alpha+\beta_{1} \mathrm{X} 1_{\mathrm{i}}+\beta_{2} \mathrm{X} 2_{\mathrm{i}}+\beta_{3} \mathrm{X} 3_{\mathrm{i}}+\beta_{4} \mathrm{X} 4_{\mathrm{i}}+\beta_{5} \mathrm{X} 5_{\mathrm{i}}+\beta_{6} \mathrm{X} 6_{\mathrm{i}}+\beta_{7} \mathrm{X} 7_{\mathrm{i}}+\beta_{8} \mathrm{X} 8_{\mathrm{i}}+\beta_{9} \mathrm{X} 9_{\mathrm{i}}+\beta_{10} \mathrm{X} 10_{\mathrm{i}}+ \\
\beta_{11} \mathrm{X} 11_{\mathrm{i}}+\beta_{12} \mathrm{X} 12_{\mathrm{i}}+\beta_{13} \mathrm{X} 13_{\mathrm{i}}+\beta_{14} \mathrm{X} 14_{\mathrm{i}}+\beta_{15} \mathrm{X} 15_{\mathrm{i}}+\beta_{16} \mathrm{X} 16_{\mathrm{i}}+\varepsilon_{\mathrm{i}}
\end{gathered}
$$

Onde:

Y - Maturidade Percebida em GP

X1 - Gerenciamento da Integração

$\mathrm{X} 2$ - Gerenciamento do Escopo

X3 - Gerenciamento do Tempo

X4 - Gerenciamento dos Custos

X5 - Gerenciamento da Qualidade

X6 - Gerenciamento dos RH

X7 - Gerenciamento das Comunicações

X8 - Gerenciamento dos Riscos

X9-Gerenciamento das Aquisições

X10 - Gerenciamento dos Stakeholders

X11 - Gênero

X12 - Tipo de servidor

X13 - Cargo de Chefia

X14 - Tempo de Instituto

X15 - Grau de Instrução

X16 - Capacitação em GP 
Parte da análise qualitativa foi realizada a partir das informações coletadas nas perguntas abertas, sendo que as barreiras e facilidades para implementação do gerenciamento de projetos, após transcritas, foram agrupadas e compiladas por temas-chaves. Em seguida foram apresentadas em formas de nuvens de palavras, utilizando-se o software Tagul, o que possibilitou a organização gráfica das expressões com maior incidência.

Realizando uma discussão acerca da análise quantitativa e qualitativa foi possível concluir a triangulação, convergir as evidências, determinar o grau em que as dez áreas do conhecimento do gerenciamento de projetos são percebidas e têm sido adotadas pelas equipes de projetos estratégicos do IFES, avaliar o atual nível de maturidade em gerenciamento de projetos do Instituto e por fim determinar os fatores que influenciam sua variação.

\section{Apresentação e análise dos resultados}

\subsection{Análise quantitativa}

Foram coletados 142 questionários de líderes e membros de equipes de projetos estratégicos, e as análises são descritas nas seções a seguir.

\subsubsection{Estatística descritiva da amostra}

Procederam-se análises estatísticas descritivas com o objetivo de identificar as áreas do conhecimento em gerenciamento de projetos que são mais aplicadas nos projetos estratégicos do IFES, conforme mostrado na Tabela 3.

Tabela 3: Aplicabilidade das áreas de conhecimento e processos - PMBOK

\begin{tabular}{lcccc}
\hline \multicolumn{1}{c}{ Área do Conhecimento } & Média & $\begin{array}{c}\text { Desvio } \\
\text { Padrão }\end{array}$ & $\begin{array}{c}\mathbf{9 5 \%} \\
\text { Inferior }\end{array}$ & $\begin{array}{c}\mathbf{9 5 \%} \\
\text { Superior }\end{array}$ \\
\hline Gerenciamento da Integração & $\mathbf{3 , 7 3}$ & $\mathbf{0 , 7 9}$ & $\mathbf{3 , 6 0}$ & $\mathbf{3 , 8 6}$ \\
\hline Desenvolvimento do termo de abertura & 4,06 & 0,90 & 3,91 & 4,21 \\
Desenvolvimento do plano de gerenciamento & 3,92 & 0,91 & 3,77 & 4,07 \\
Direção e gerenciamento da execução & 3,95 & 0,88 & 3,81 & 4,09 \\
Monitoramento e controle do trabalho & 3,67 & 1,02 & 3,50 & 3,84 \\
Avaliação de solicitação de mudança & 3,39 & 1,03 & 3,22 & 3,56 \\
Fechamento do projeto & 3,39 & 1,06 & 3,22 & 3,56 \\
\hline Gerenciamento do Escopo & $\mathbf{3 , 5 9}$ & $\mathbf{0 , 8 9}$ & $\mathbf{3 , 4 4}$ & $\mathbf{3 , 7 4}$ \\
\hline Definição do Escopo & 3,92 & 0,93 & 3,77 & 4,07 \\
Criação da EAP (Estrutura Analítica do Projeto) & 3,61 & 0,99 & 3,45 & 3,77 \\
Verificação formal da execução do escopo & 3,39 & 1,09 & 3,21 & 3,57 \\
Monitoramento e Controle das mudanças no escopo & 3,44 & 1,01 & 3,27 & 3,61 \\
\hline Gerenciamento do Tempo & $\mathbf{3 , 9 0}$ & $\mathbf{0 , 7 3}$ & $\mathbf{3 , 7 8}$ & $\mathbf{4 , 0 2}$ \\
\hline Identificação de atividades para executar & 4,15 & 0,80 & 4,02 & 4,28 \\
Sequenciamento de atividades & 4,08 & 0,82 & 3,95 & 4,21 \\
Identificação de recursos necessários & 3,93 & 0,80 & 3,80 & 4,06 \\
Estimativa de duração das atividades & 3,84 & 0,83 & 3,70 & 3,98 \\
Desenvolvimento de cronograma & 3,80 & 0,96 & 3,64 & 3,96 \\
\hline
\end{tabular}




\begin{tabular}{|c|c|c|c|c|}
\hline Área do Conhecimento & Média & $\begin{array}{l}\text { Desvio } \\
\text { Padrão }\end{array}$ & $\begin{array}{c}95 \% \\
\text { Inferior }\end{array}$ & $\begin{array}{c}95 \% \\
\text { Superior }\end{array}$ \\
\hline Monitoramento e controle do cronograma & 3,58 & 0,98 & 3,42 & 3,74 \\
\hline Gerenciamento dos Custos & 3,33 & 1,00 & 3,17 & 3,49 \\
\hline Estimativa de custos & 3,40 & 1,01 & 3,23 & 3,57 \\
\hline Desenvolvimento de orçamento & 3,34 & 1,03 & 3,17 & 3,51 \\
\hline Monitoramento e controle do orçamento & 3,26 & 1,05 & 3,09 & 3,43 \\
\hline Gerenciamento da Qualidade & 3,26 & 0,95 & 3,10 & 3,42 \\
\hline Identificação dos requisitos de qualidade & 3,39 & 1,00 & 3,23 & 3,55 \\
\hline Realização de auditorias de qualidade & 3,09 & 1,01 & 2,92 & 3,26 \\
\hline Monitoramento e controle da qualidade & 3,28 & 1,01 & 3,11 & 3,45 \\
\hline Gerenciamento dos Recursos Humanos & 3,75 & 0,94 & 3,60 & 3,90 \\
\hline Alocação racional de servidores à equipe & 3,84 & 0,98 & 3,68 & 4,00 \\
\hline Desenvolvimento da equipe & 3,72 & 1,05 & 3,55 & 3,89 \\
\hline Gerenciamento da equipe & 3,66 & 1,03 & 3,49 & 3,83 \\
\hline Gerenciamento das Partes Interessadas & 3,76 & $\mathbf{0 , 8 4}$ & 3,62 & 3,90 \\
\hline Identificação das partes interessadas & 3,95 & 0,84 & 3,81 & 4,09 \\
\hline Coleta de requisitos das partes interessadas & 3,69 & 0,93 & 3,54 & 3,84 \\
\hline Gerenciamento das partes interessadas & 3,63 & 0,99 & 3,47 & 3,79 \\
\hline Gerenciamento das Comunicações & $\mathbf{3 , 5 0}$ & 0,92 & 3,35 & 3,65 \\
\hline Desenvolvimento do plano de comunicação & 3,56 & 1,04 & 3,39 & 3,73 \\
\hline Disponibilidade da informação a todos interessados & 3,58 & 0,96 & 3,42 & 3,74 \\
\hline Recolhimento e distribuição de relatórios & 3,37 & 1,02 & 3,20 & 3,54 \\
\hline Gerenciamento dos Riscos & 2,95 & 0,99 & 2,79 & 3,11 \\
\hline Desenvolvimento de um plano de riscos & 2,88 & 1,00 & 2,72 & 3,04 \\
\hline Identificação dos riscos & 3,07 & 1,01 & 2,90 & 3,24 \\
\hline Realização de análise quali/quanti dos riscos & 2,87 & 1,11 & 2,69 & 3,05 \\
\hline Desenvolvimento de planos de resposta aos riscos & 2,94 & 1,01 & 2,77 & 3,11 \\
\hline Monitoramento e controle dos riscos & 2,99 & 1,06 & 2,82 & 3,16 \\
\hline Gerenciamento das Aquisições & 3,41 & 0,92 & 3,26 & 3,56 \\
\hline Documentação de aquisições/contratações & 3,46 & 0,93 & 3,31 & 3,61 \\
\hline Gerenciamento de contratos & 3,41 & 0,95 & 3,25 & 3,57 \\
\hline Verificação de todos os contratos & 3,37 & 0,94 & 3,22 & 3,52 \\
\hline
\end{tabular}

Fonte: Elaborado pelos autores.

De forma complementar, foram levantados dados sobre a importância percebida pelos líderes e equipes dos projetos estratégicos em relação às áreas do conhecimento, conforme Tabela 4 .

Tabela 4: Importância percebida das áreas do conhecimento

\begin{tabular}{lcccc}
\hline \multicolumn{1}{c}{ Área do Conhecimento } & Média & $\begin{array}{c}\text { Desvio } \\
\text { Padrão }\end{array}$ & $\begin{array}{c}\mathbf{9 5 \%} \\
\text { Inferior }\end{array}$ & $\begin{array}{c}\mathbf{9 5 \%} \\
\text { Superior }\end{array}$ \\
\hline Gerenciamento da Integração & 4,75 & 0,46 & 4,674 & 4,826 \\
\hline Gerenciamento do Escopo & 4,61 & 0,52 & 4,524 & 4,696 \\
\hline
\end{tabular}




\begin{tabular}{lllll}
\hline Gerenciamento do Tempo & 4,46 & 0,60 & 4,361 & 4,559 \\
\hline Gerenciamento dos Custos & 4,54 & 0,58 & 4,445 & 4,635 \\
\hline Gerenciamento da Qualidade & 4,80 & 0,42 & 4,731 & 4,869 \\
\hline Gerenciamento dos Recursos Humanos & 4,77 & 0,44 & 4,698 & 4,842 \\
\hline Gerenciamento das Partes Interessadas & 4,69 & 0,51 & 4,606 & 4,774 \\
\hline Gerenciamento das Comunicações & 4,79 & 0,41 & 4,723 & 4,857 \\
\hline Gerenciamento dos Riscos & 4,41 & 0,57 & 4,316 & 4,504 \\
\hline Gerenciamento das Aquisições & 4,29 & 0,72 & 4,172 & 4,408 \\
\hline
\end{tabular}

Fonte: Elaborado pelos autores.

Percebe-se que todas as áreas de conhecimento tiveram médias superiores a 4 , tendendo a concordar fortemente a importância de cada uma.

Para comparar estatisticamente as médias de aplicabilidade e importância para cada área do conhecimento foi aplicado o teste t para diferença de médias, os resultados são mostrados na Tabela 5 .

Tabela 5: Teste $t$ para diferenças de médias

\begin{tabular}{|c|c|c|c|c|c|c|}
\hline Áreas do conhecimento & Média & $\begin{array}{l}\text { Desvio } \\
\text { Padrão }\end{array}$ & $\begin{array}{l}\text { Erro padrão } \\
\text { da média }\end{array}$ & $\mathrm{t}$ & $\begin{array}{c}\text { Sig. (2 } \\
\text { extremi- } \\
\text { dades) }\end{array}$ & $\begin{array}{c}\text { Diferença } \\
\text { entre as } \\
\text { médias }\end{array}$ \\
\hline \multirow{2}{*}{ Integração } & 3,73 & 0,79 & 0,66 & \multirow{2}{*}{$-13,620$} & \multirow{2}{*}{0,00} & \multirow{2}{*}{$-1,021$} \\
\hline & 4,75 & 0,46 & 0,39 & & & \\
\hline \multirow{2}{*}{ Escopo } & 3,59 & 0,88 & 0,74 & \multirow{2}{*}{$-11,834$} & \multirow{2}{*}{0,00} & \multirow{2}{*}{$-1,007$} \\
\hline & 4,61 & 0,52 & 0,43 & & & \\
\hline \multirow[b]{2}{*}{ Tempo } & 3,90 & 0,73 & 0,61 & \multirow[b]{2}{*}{$-7,966$} & \multirow[b]{2}{*}{0,00} & \multirow[b]{2}{*}{$-0,557$} \\
\hline & 4,46 & 0,60 & 0,51 & & & \\
\hline \multirow[b]{2}{*}{ Custos } & 3,33 & 1,00 & 0,84 & \multirow{2}{*}{$-13,191$} & \multirow[b]{2}{*}{0,00} & \multirow{2}{*}{$-1,202$} \\
\hline & 4,54 & 0,58 & 0,49 & & & \\
\hline \multirow[b]{2}{*}{ Qualidade } & 3,26 & 0,95 & 0,80 & \multirow[b]{2}{*}{$-17,664$} & \multirow[b]{2}{*}{0,00} & \multirow[b]{2}{*}{$-1,540$} \\
\hline & 4,80 & 0,42 & 0,35 & & & \\
\hline \multirow{2}{*}{ Recursos Humanos } & 3,75 & 0,94 & 0,79 & \multirow{2}{*}{$-12,052$} & \multirow{2}{*}{0,00} & \multirow{2}{*}{$-1,025$} \\
\hline & 4,77 & 0,44 & 0,37 & & & \\
\hline \multirow{2}{*}{ Partes Interessadas } & 3,76 & 0,84 & 0,71 & \multirow{2}{*}{$-11,099$} & \multirow{2}{*}{0,00} & \multirow{2}{*}{$-0,933$} \\
\hline & 4,69 & 0,51 & 0,43 & & & \\
\hline \multirow{2}{*}{ Comunicação } & 3,50 & 0,92 & 0,77 & \multirow{2}{*}{$-16,032$} & \multirow{2}{*}{0,00} & \multirow{2}{*}{$-1,284$} \\
\hline & 4,79 & 0,41 & 0,34 & & & \\
\hline
\end{tabular}




\begin{tabular}{|c|c|c|c|c|c|c|}
\hline \multirow{2}{*}{ Riscos } & 2,95 & 0,99 & 0,83 & \multirow{2}{*}{$-15,459$} & \multirow{2}{*}{0,00} & \multirow{2}{*}{$-1,459$} \\
\hline & 4,41 & 0,57 & 0,48 & & & \\
\hline \multirow{2}{*}{ Aquisições } & 3,41 & 0,92 & 0,77 & \multirow{2}{*}{$-9,825$} & \multirow{2}{*}{0,00} & \multirow{2}{*}{$-0,878$} \\
\hline & 4,29 & 0,72 & 0,60 & & & \\
\hline
\end{tabular}

Fonte: Elaborado pelos autores.

A análise realizada indica que para todas as áreas do conhecimento há diferença significativa entre as médias de aplicabilidade e importância percebida, sendo os $\mathrm{p}$ valores $<0,05$. Em todos os casos a média da importância percebida da área foi maior que a sua aplicabilidade, isto é evidenciado pelo sinal negativo da diferença entre as médias (Média Aplicabilidade - Média Importância). Os respondentes informam dar consideravelmente mais importância nessas áreas do que de fato implementam-nas nos projetos em que fazem parte, isso mostra um caminho para o Escritório de Gerenciamento de Projetos (EGP) do IFES trabalhar para reduzir essa lacuna.

Analisando descritivamente a variável dependente Maturidade em Gerenciamento de Projetos Percebida, temos a seguinte situação conforme Tabela 6 .

Tabela 6: Estatística descritiva da variável dependente

\begin{tabular}{|c|c|c|c|c|}
\hline & & & Estatística & Erro Padrão \\
\hline \multirow{11}{*}{$\begin{array}{c}\text { IFES - Nível de } \\
\text { Maturidade em } \\
\text { GP }\end{array}$} & \multicolumn{2}{|l|}{ Média } & 2,37 & 0,09 \\
\hline & \multirow{2}{*}{$\begin{array}{l}\text { Intervalo de confiança a } 95 \% \text { para a } \\
\text { média }\end{array}$} & Limite Inferior & 2,19 & \\
\hline & & Limite Superior & 2,55 & \\
\hline & \multicolumn{2}{|c|}{ Média ajustada a $5 \%$} & 2,34 & \\
\hline & \multicolumn{2}{|l|}{ Mediana } & 2 & \\
\hline & \multicolumn{2}{|c|}{ Desvio padrão } & 1,08 & \\
\hline & \multicolumn{2}{|l|}{ Mínimo } & 1 & \\
\hline & \multicolumn{2}{|l|}{ Máximo } & 5 & \\
\hline & \multicolumn{2}{|l|}{ Amplitude } & 4 & \\
\hline & \multicolumn{2}{|l|}{ Assimetria } & 0,32 & 0,20 \\
\hline & \multicolumn{2}{|l|}{ Curtose } & $-1,04$ & \\
\hline
\end{tabular}

Fonte: Elaborado pelos autores.

A média da maturidade percebida em GP do IFES $(M=2,37)$ com desvio padrão 1,08 assegura o limite inferior $(2,19)$ e limite superior $(2,55)$ a $95 \%$ de confiança, o que indica que a maturidade esteja entre os níveis Processos e Padrões Estruturados (Nível 2) e Padrões Organizacionais Institucionalizados (Nível 3).

De fato, os dados levantados certificam a realidade institucional. Apenas recentemente o EGP do IFES entregou a estruturação dos processos de gerenciamento de projetos e alguns projetos já seguem tal metodologia, isso explica porque a maturidade de nível 3 não seja atingida plenamente. Os dados evidenciam que há uma grande oportunidade para melhorar as práticas de gerenciamento de projetos dentro do Instituto. O próximo desafio do PMO é institucionalizar os padrões à aplicação em todos os projetos estratégicos, coletar informalmente a aplicação por meio de auditorias e obter análise ainda que informal do desempenho dos projetos. Dessa forma, o IFES conseguirá prosseguir para um nível mais alto de maturidade. 


\subsubsection{Regressão linear múltipla}

A estimação das variáveis foi dada pelo método stepwise e o resumo da análise do modelo gerado é mostrado na Tabela 7.

Tabela 7: Resumo do modelo

\begin{tabular}{|c|c|c|c|c|c|c|c|c|c|}
\hline \multirow{2}{*}{ Modelo } & \multirow{2}{*}{$\mathrm{R}$} & \multirow{2}{*}{$\mathrm{R}^{2}$} & \multirow{2}{*}{$\begin{array}{c}\mathrm{R}^{2} \\
\text { Ajustado }\end{array}$} & \multirow{2}{*}{$\begin{array}{l}\text { Erro padrão } \\
\text { do estimador }\end{array}$} & \multicolumn{5}{|c|}{ Estatísticas de Mudança } \\
\hline & & & & & Variação $\mathrm{R}^{2}$ & Iteração F & df1 & $\mathrm{df} 2$ & Sig. F \\
\hline 6 & ,635 & ,404 & ,377 &, 854 & 0,20 & 4,554 & 1 & 135 & 035 \\
\hline
\end{tabular}

Preditores: (Constant), Gerenciamento das Aquisições, Gerenciamento do Tempo, Gerenciamento dos Riscos, Gerenciamento das Comunicações, Gerenciamento dos Custos, Grau de Instrução.

Variável Dependente: IFES/Nível de Maturidade em Gerenciamento de Projetos

Fonte: Elaborado pelos autores

A qualidade do modelo 6 pode ser verificada pelo coeficiente de determinação de $\mathrm{R}^{2}$ ajustado, no qual podemos afirmar que as variáveis independentes explicam $37,7 \%$ da variação da variável dependente Nível de Maturidade Percebido em GP do IFES, sendo que o restante dessa variação é explicado por outros fatores.

A ANOVA da regressão linear, teste empregado para verificar a significância do modelo como um todo foi suportada, sendo F significante com valor $\mathrm{P}<0.001$. Também foi analisada a aderência da variável dependente à distribuição normal, o uso de variáveis categóricas (Likert) nas teorias baseadas em distribuições normais devem ser utilizadas apenas quando a variável dependente apresentar baixos valores de assimetria entre -1 e +1 , e curtose entre $-1,5$ e $+1,5$ (valores próximos das distribuições normais) permitindo a aplicação de testes paramétricos apenas sob tais condições (Schumacker \& Lomax, 2004). No modelo, a assimetria e a curtose da curva gerada são 0,32 e -1,04, respectivamente, aceitando os requisitos de aproximação da normalidade, permitindo que se aplique testes paramétricos no modelo gerado.

O teste de runs não rejeitou a hipótese nula da distribuição de valores das frequências de forma aleatória, com pvalor Asymp.Sig $>0,05$. O resultado do pvalor no teste Breusch-Pagan foi de $0,3479>0,05$, sendo a probabilidade do teste maior que $5 \%$, não se pode rejeitar a hipótese nula da homocedasticidade, ou seja, condicional às variáveis explicativas a variância do erro no modelo é constante.

Após esta série de testes estatísticos é possível a construção da Tabela 8 que apresenta os resultados dos níveis de significância da regressão linear e os coeficientes das estimativas dos 6 fatores que foram significativos a $95 \%$ de confiança. A regressão mostrou que as variáveis que se relacionaram significativamente à variável dependente Maturidade Percebida em Gerenciamento de Projetos foram: Gerenciamento das Aquisições, Gerenciamento do Tempo, Gerenciamento dos Riscos, Gerenciamento da Comunicação, Gerenciamento dos Custos e Grau de Instrução. 
Tabela 8: Coeficientes estimados

\begin{tabular}{|c|c|c|c|c|c|c|c|c|}
\hline & \multirow{2}{*}{ Modelo - Variáveis } & \multicolumn{2}{|c|}{$\begin{array}{c}\text { Coeficientes não } \\
\text { padronizados }\end{array}$} & \multirow{2}{*}{$\begin{array}{c}\begin{array}{c}\text { Coeficientes } \\
\text { padronizados }\end{array} \\
\text { Beta }\end{array}$} & \multirow{2}{*}{$\mathbf{t}$} & \multirow{2}{*}{ Sig. } & \multicolumn{2}{|c|}{$\begin{array}{l}\text { Estatística de } \\
\text { colinearidade }\end{array}$} \\
\hline & & B & Erro padrão & & & & Tolerância & VIF \\
\hline \multirow{7}{*}{6} & (Constante) & $-0,10$ & 0,525 & & $-0,019$ & 0,985 & & \\
\hline & $\begin{array}{l}\text { Gerenciamento das } \\
\text { Aquisições }\end{array}$ & 0,306 & 0,112 & 0,260 & 2,726 & 0,007 & 0,487 & 2,054 \\
\hline & $\begin{array}{l}\text { Gerenciamento do } \\
\text { Tempo }\end{array}$ & 0,256 & 0,120 & 0,173 & 2,140 & 0,034 & 0,672 & 1,488 \\
\hline & $\begin{array}{l}\text { Gerenciamento dos } \\
\text { Riscos }\end{array}$ & $-0,404$ & 0,099 & $-0,369$ & $-4,078$ & 0,000 & 0,541 & 1,849 \\
\hline & $\begin{array}{l}\text { Gerenciamento da } \\
\text { Comunicação }\end{array}$ & 0,366 & 0,116 & 0,312 & 3,146 & 0,002 & 0,449 & 2,228 \\
\hline & $\begin{array}{l}\text { Gerenciamento dos } \\
\text { Custos }\end{array}$ & 0,249 & 0,102 & 0,230 & 2,432 & 0,016 & 0,496 & 2,018 \\
\hline & Grau de Instrução & $-0,165$ & 0,077 & $-0,144$ & $-2,134$ & 0,035 & 0,966 & 1,035 \\
\hline
\end{tabular}

Fonte: Elaborado pelos autores.

\subsection{Análise Qualitativa}

A partir das perguntas abertas, foram analisadas as barreiras e facilidades para implementação dos projetos estratégicos. Os líderes e membros das equipes relataram abertamente suas opiniões, que foram transcritas. Em seguida, após análise de similaridade, as palavras-chaves foram agrupadas, compiladas e apresentadas em nuvens de palavras, utilizando o software Tagul, formando um quadro que possibilita a organização gráfica das expressões com maior incidência. A Figura 1 exibe essa disposição para o caso das barreiras à implementação dos projetos.

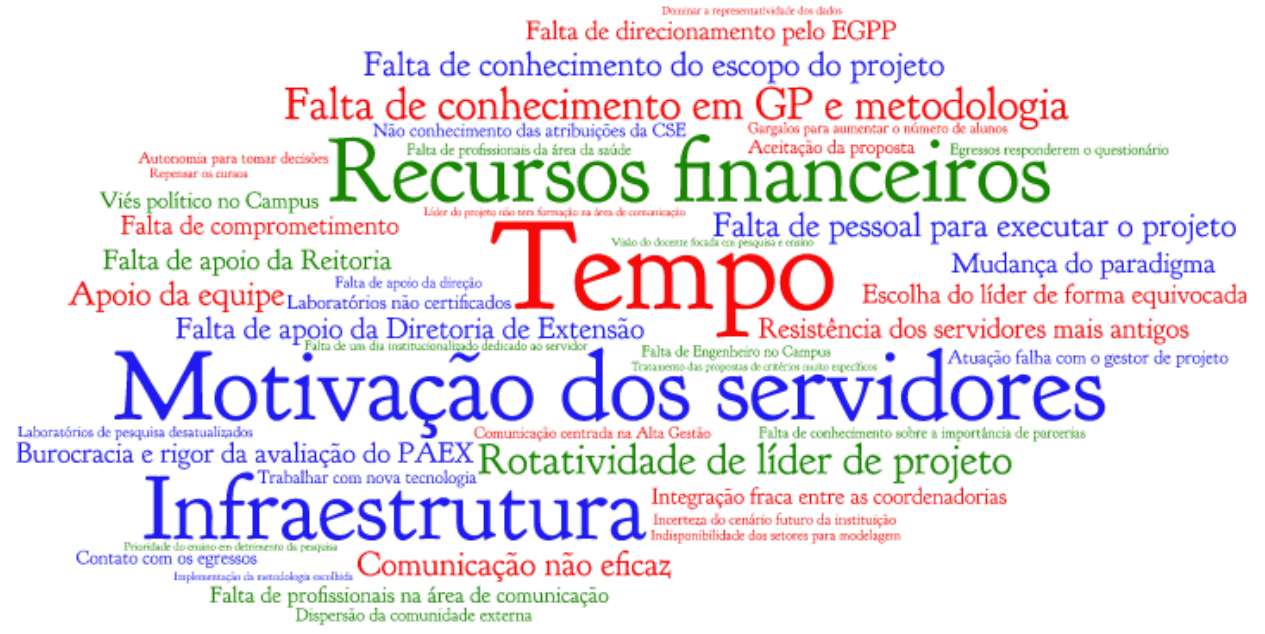

Figura 1: Barreiras para implementação dos projetos

Fonte: Elaborado pelos autores. 
As principais dificuldades relatadas pelos respondentes foram: falta de tempo para se dedicarem ao projeto, motivação dos servidores que não fazem parte da equipe em contribuir, recursos financeiros, infraestrutura inadequada e falta de conhecimento em GP e metodologia do PMO.

Por outro lado, foram levantados os fatores que facilitam a implementação dos projetos dentro do IFES, sendo representados na Figura 2.

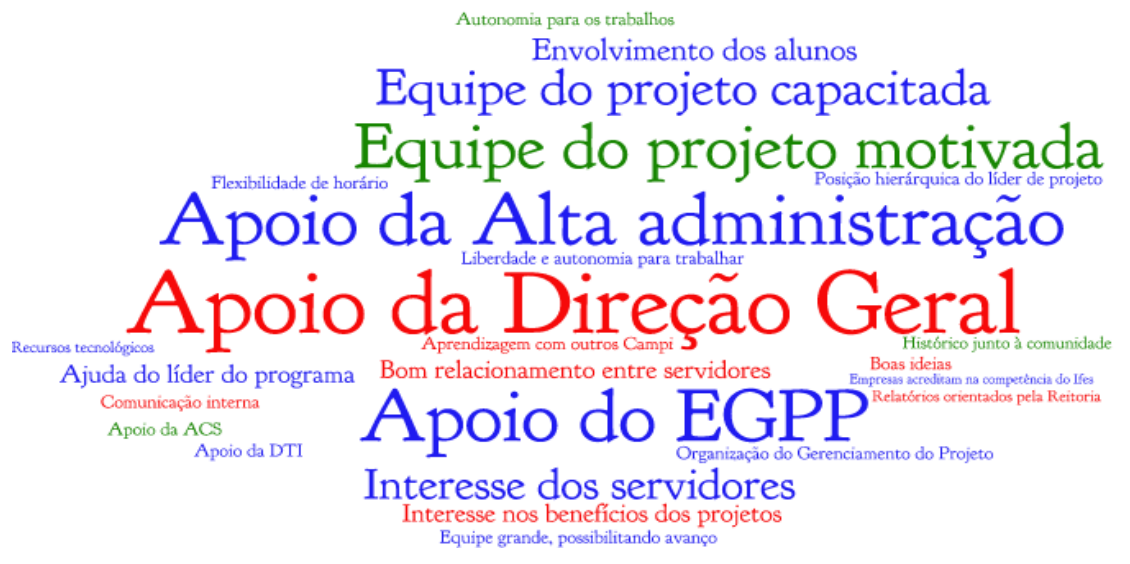

Figura 2: Facilidades para implementação dos projetos

Fonte: Elaborado pelos autores.

Destacam-se 3 principais fatores: o Apoio da Direção Geral dos Campi, o apoio da Alta Administração (Reitor, Pró-Reitor e Diretores Sistêmicos) e o apoio do EGPP (Escritório de Gerenciamento de Projetos e Processos). A equipe do projeto motivada e capacitada também aparecem em destaque como facilidades para os objetivos dos projetos serem alcançados.

\section{Discussão}

A partir da análise quantitativa ficou comprovado que as dimensões de Gerenciamento do Tempo, Gerenciamento dos Custos, Gerenciamento das Comunicações e Gerenciamento das Aquisições possuem relação positiva com a percepção de maturidade institucional em gerenciamento de projetos.

O gerenciamento do tempo do projeto engloba os processos cruciais para coordenar a finalização pontual do projeto. A definição e o sequenciamento das atividades para realização das entregas, e as estimativas de recursos e duração das atividades são fundamentais para o desenvolvimento de um cronograma exequível. O gerenciamento dos custos compreende os processos de planejamento, previsão, criação de orçamento e controle dos custos para que o projeto seja capaz de ser concluído dentro do orçamento aprovado. Portanto, o sucesso em um projeto está relacionado à manutenção de integridade das linhas de base de tempo e custo, ou seja, conservação do planejamento do cronograma e do orçamento do projeto durante a execução de um projeto (Cooke-Davies, 2002).

O gerenciamento das comunicações integra os processos essenciais para proporcionar que as informações do projeto sejam planejadas, emitidas, mantidas, resgatadas, fiscalizadas e dispostas de maneira compatível e adequada a todas as partes interessadas. Os canais de comunicação adequados e a capacidade de feedback e respostas aos clientes são fatores determinantes para o sucesso no gerenciamento dos projetos (Baker, Murphy, \& Fisher, 1988; Cleland \& King, 1983; Lock, 1984). 
O gerenciamento das aquisições do projeto compreende os processos essenciais para comprar ou adquirir produtos, serviços ou resultados externos à equipe do projeto. Alcançar excelência na gestão de contratos é de fundamental importância para o cumprimento das obrigações contratuais e um relacionamento confiável com fornecedores da administração pública. O papel dos contratos e o desenvolvimento de relacionamentos são considerados fatores importantes para a compreensão da gestão e organização de projetos, tendo o gerenciamento de aquisições, por meio de contratos, considerado fator de sucesso (como) de um projeto (Abdullah \& Ramly, 2006).

A partir da análise qualitativa ficou evidenciado que as dimensões Estrutura Organizacional e Gerenciamento das Partes Interessadas possuem relação positiva com a percepção de maturidade institucional em gerenciamento de projetos.

A estrutura organizacional funcional presente no Instituto não facilita o gerenciamento dos projetos, consequentemente a implementação de seu planejamento estratégico. Esta estrutura tende a ser orientada às suas atividades rotineiras, existindo nela uma tendência a subestimar os projetos estratégicos. O IFES pode migrar para estruturas mais flexíveis e aderentes ao gerenciamento de projetos tais como estrutura orientada a projetos ou matricial, sendo uma proposta mais adequada para o atual momento do Instituto que visa a transformação organizacional através da execução de projetos, uma vez que, há relação entre gerente de projeto dedicado e o atendimento do cronograma do projeto (Berssaneti \& Carvalho, 2015).

Para aplicação do gerenciamento das partes interessadas sugere-se ao Instituto identificar os grupos de pessoas ou organizações que podem impactar ou serem impactadas pelo projeto, analisar suas expectativas e desenvolver estratégias de engajamento destas expectativas por meio das entregas, à procura de alcançar plenamente o objetivo do projeto. A Alta Administração pode focar em ser vista como a liderança para motivar a consciência dos colaboradores em ver projetos como um veículo para o gerenciamento de todos os tipos de mudança. Desta forma, pode garantir que o sistema de gerenciamento de projetos apoie o desenvolvimento da uma cultura de projetos como transformadores organizacionais (Qureshi, Warraich, \& Hijazi, 2009), uma vez que existe uma relação significativa entre a cultura organizacional e desempenho dos projetos (Yazici, 2009).

\section{Considerações finais}

Os resultados mostram que, em todas as áreas do conhecimento do PMBOK (PMI, 2013), os membros das equipes dos projetos informam dar consideravelmente mais importância do que propriamente aplicam-nas nos projetos em que fazem parte. Esta evidência demonstra uma possibilidade para o EGP do IFES aumentar a maturidade institucional no gerenciamento de seus projetos, ou seja, trabalhar para reduzir esta lacuna entre aplicabilidade e importância percebida é motivar a utilização de boas práticas reconhecidas e assim aumentar a maturidade institucional, adquirindo maior sucesso no gerenciamento de seus empreendimentos.

Novamente, ressalte-se que, para fins desta pesquisa, embora uma sexta versão do PMBOK já esteja disponível a partir de 2017, o objeto de estudo em questão, o IFES, utiliza como base a quinta versão, de 2013, inclusive tendo sido essa a base do treinamento dos gestores de projetos e da metodologia de projetos elaborada e implementada na Instituição.

$\mathrm{Na}$ escala de 1 a 5 do PMMM, o atual nível de maturidade em gerenciamento de projetos percebido no Instituto foi 2,37, estando entre os níveis Processos e Padrões Estruturados e Padrões Organizacionais Institucionalizados, tendendo ao primeiro. Para desenvolver-se e alcançar plenamente o nível 3 de maturidade o IFES necessita padronizar os processos de gerenciamento de projetos e institucionalizar a prática a todos os projetos estratégicos, coletar informalmente a 
aplicação por meio de auditorias e obter análise ainda que informal do desempenho dos projetos.

Assim, o IFES pode concentrar esforços para melhorar as suas capacidades, desenvolvendo-se para níveis de maturidade mais elevados nas áreas do conhecimento determinantes, são elas: o gerenciamento do tempo, custos, comunicações, partes interessadas, aquisições, o grau de instrução do profissional, e a estrutura organizacional com um gerente de projeto mais dedicado e apoio da alta administração (Diretor Geral, Reitoria e PMO).

Portanto, os resultados do estudo permitem o direcionamento de políticas e incentivos para aumentar o desempenho de projetos estratégicos do Instituto Federal, gerenciando melhor a utilização de recursos públicos, elevando o grau de sucesso dos empreendimentos, oferecendo maior transparência e facilidade na construção do relatório de gestão, fortalecendo e racionalizando as ações de controle e minimizando a ocorrência de improbidades e irregularidades no processo de gestão, facilitando a accountability por parte da governança no cumprimento de sua missão institucional: "Promover educação profissional pública de excelência, integrando ensino, pesquisa e extensão, para a construção de uma sociedade democrática, justa e sustentável”.

Como limitante desta pesquisa, destacamos que apenas os projetos estratégicos foram analisados. Entretanto, o IFES trabalha com outras naturezas de projetos, tais como: projetos de pesquisa, projetos de desenvolvimento de produtos e serviços, projetos de ensino e projetos de trabalho que visam desenvolvimento de competências específicas. Além disso, por ter sido um estudo de um único caso, há que destacar que os resultados deste estudo não podem ser generalizados para instituições de ensino superior, tampouco para instituições públicas.

Assim, pesquisas futuras poderão contribuir para a temática pesquisada, abordando projetos de natureza não apenas estratégica, assim como trabalhar com outras variáveis de ordem pessoal, de motivacional e de liderança. A realização de pesquisas como esta em outros Institutos Federais e outras instituições de ensino públicas ou privadas poderá trazer subsídios complementares e ampliar, confirmar ou mesmo contradizer as conclusões do presente estudo, contribuindo para o campo do gerenciamento de projetos no segmento educacional.

Em suma, embora os resultados não possam ser generalizados, indicam caminhos para o direcionamento de políticas e incentivos para aumentar o desempenho de projetos estratégicos em instituições públicas de educação.

\section{Referências}

Abdullah, W. M. W. \& Ramly, A. (2006). Does successful project management equates to project success? Proceedings of $5^{\text {th }}$ IEEE International Conference of Cognitive Informatics 2006, Beijing, China. Recuperado em 29 de janeiro, 2019, de http://eprints.utm.my/id/eprint/648/1/CM_53\%5B1\%5D._Does_Successful_PM._Wan_Maimun.pdf.

Baker, B. N., Murphy, D. C., \& Fisher, D. (1988). Factors affecting project success. In: Cleland, D. I. \& King, W. R. (Eds.). Project Management Handbook. New York: John Wiley \& Sons, Inc.

Berssaneti, F. T. \& Carvalho, M. M. (2015). Identification of variables that impact project success in Brazilian companies. International Journal of Project Management, 33(3), 638-649.

Bryde, D. J. (2003). Modelling project management performance. International Journal of Quality \& Reliability Management, 20(2), 229-254.

Carvalho, M. M., Patah, L. A., \& Bido, D. S. (2015). Project management and its effects on project success: Cross-country and cross-industry comparisons. International Journal of Project Management, 33(7), 1509-1522. 
Cleland, D. I. \& King, W. R. (1983). System analysis and project management. New York: McGraw-Hill.

Crawford, J. K. (2001). Project management maturity model: Providing a proven path to project management excellence. New York: CRC Press.

Crawford, L. H. \& Helm, J. (2009). Government and governance: the value of project management in the public sector. Project Management Journal, 40(1), 73-87.

Cooke-Davies, T. (2002). The 'real' success factors on projects. International Journal of Project Management, 20(3), 185-190.

Cooke-Davies, T. (2004). Project management maturity models. In: Moris, P. W. G. \& Pinto, J. K. (Eds.). The Wiley guide to managing projects. Hoboken, New Jersey: John Wiley \& Sons.

Cooke-Davies, T. \& Arzymanow, A. (2003). The maturity of project management in different industries: An investigation into variations between project management models. International Journal of Project Management, 21(6), 471-478.

Eisenhardt, K. M. (1989). Building theories from case study research. Academy of Management Review, 14(4), 532-550.

Edwards, M. \& Clough, R. (2005). Corporate governance and performance: an exploration of the connection in a public sector context. Canberra: University of Canberra.

Görög, M. (2016). A broader approach to organisational project management maturity assessment. International Journal of Project Management, 34(8), 1658-1669.

Grant, K. P. \& Pennypacker, J. S. (2006). Project management maturity: an assessment of project management capabilities among and between selected industries. IEEE Transactions on Engineering Management, 53(1), 59-68.

Ibbs, W. C. \& Kwak, Y. H. (2000). Assessing project management maturity. Project Management Journal, 31(1), 32-43.

Ika, L., Diallo, A., \& Thuillier, D. (2012). Critical success factors for World Bank projects: An empirical investigation. International Journal of Project Management, 30(1), 105-116.

Kerzner, H. \& Kerzner, H. R. (2017). Project management: a systems approach to planning, scheduling, and controlling. Hoboken, New Jersey: Willey \& Sons.

Lock, D. (1984). Project management. New York: St. Martins Press.

Meng, X., Sun, M., \& Jones, M. (2011). Maturity model for supply chain relationships in construction. Journal of Management in Engineering, 27(2), 97-105.

Mullaly, M. (2014). If maturity is the answer, then exactly what is the question? International Journal of Managing Projects in Business, 7(2), 169-185.

Neves, L. A. L., Trentim, M. H., \& Nunes, L. N. P. (2014). Estudo de caso sobre maturidade e sucesso em uma Instituição de ciência e tecnologia do Governo Federal. Recuperado em 29 de janeiro, 2019, de https://pmkb.com.br/artigos/estudo-de-caso-sobre-maturidade-e-sucesso-em-uma-instituicao-de-cienciae-tecnologia-do-governo-federal/.

Pasian, B.L., Williams, N., \& Alameri, H. (2012). The value of project management maturity models: a new conceptual model with a resource-based view. Proceedings of 26th IPMA World Congress, Crete, Greece.

PMI - Project Management Institute. (2013). A guide to the project management body of knowledge (PMBOK). $5^{\text {th }}$ Edition. Pennsylvania: PMI Publications, 2013. 
Prado, D., \& Archibald, R. (2009). Pesquisa sobre maturidade em gerenciamento de projetos - Maturidade Brasil 2008. Recuperado em 13 de abril, 2018, de http://www.maturityresearch.com.

Qureshi, T. M., Warraich, A. S., \& Hijazi, S. T. (2009). Significance of project management performance assessment (PMPA) model. International Journal of Project Management, 27(4), 378-388.

Rasid, A. S., Ismail, W., Mohammad, N., \& Long, C. (2014). Assessing adoption of project management knowledge areas and maturity level: Case study of a public agency in Malaysia. Journal of Management in Engineering, 30(2), 264-271.

Rea, L. M. \& Parker, R. A. (2000). Metodologia de pesquisa: do planejamento à execução. São Paulo: Pioneira.

Remenyi, D., Willams, B., Money, A., \& Swartz, E. (1998). Doing research in business and management: an introduction to process and method. London: Sage Publications.

Schumacker, R. E. \& Lomax, R. G. (2004). A beginner's guide to structural equation modeling. $2^{\text {nd }}$ Edition. New Jersey: Lawrence Erlbaum Associates, Inc.

Srivannaboon, S. (2006). 2006 Graduate student of the year award winner: Linking project management with business strategy. Project Management Journal, 37(5), 88-96.

Wheatley, M. (2007). Maturity matters. PM Network, 21(7), 49-53.

Yazici, H. J. (2009). The role of project management maturity and organizational culture in perceived performance. Project Management Journal, 40(3), 14-33.

Yin, R. K. (2015). Estudo de caso: planejamento e métodos. $5^{\text {a }}$ ed. São Paulo: Bookman. 